\title{
A clinical study on the changing dynamics of disease severity, management strategies and outcomes of COVID-19 in patients requiring haemodialysis
}

\author{
Govindasamy Nithya ${ }^{1} \cdot$ Tanuj Moses Lamech $^{1}\left[\right.$. Venkatesh Arumugam ${ }^{1} \cdot$ Thanikachalam Dineshkumar $^{1}$. \\ Natarajan Gopalakrishnan ${ }^{1}$. Dhanapalan Aiswarya ${ }^{1}$ - Sajmi Shaji ${ }^{1}$ - Bhagavatula V. R. H. Sastry ${ }^{1}$. Dolphin Solomon ${ }^{1}$. \\ Badri Srinivasan Kannan ${ }^{1} \cdot$ Ramanathan Sakthirajan ${ }^{1} \cdot$ Padmaraj Rajendran ${ }^{1}$
}

Received: 3 March 2021 / Accepted: 9 May 2021 / Published online: 29 May 2021

(c) Italian Society of Nephrology 2021

\begin{abstract}
Introduction In this observational study, we describe the change in the clinical profile and outcome of Corona Virus Disease 2019 (COVID-19) over the course of the outbreak, among patients requiring dialysis, including chronic haemodialysis therapy.

Methods This is a single-centre prospective observational study of patients with COVID-19 (as confirmed by RT-PCR) and renal failure requiring haemodialysis. Their clinical profiles and outcomes were analysed, vis-à-vis the changing disease severity.

Findings A total of 483 patients were included, of whom 416 had end-stage renal disease and were on maintenance haemodialysis. Patients who were symptomatic at presentation had significantly higher levels of Neutrophil-lymphocyte ratio (NLR) $(p<0.001)$, C-reactive protein (CRP) $(p<0.001)$, lactate dehydrogenase (LDH) $(p<0.001)$, higher degrees of lung involvement $(p<0.001)$ and required more respiratory support $(p<0.001)$. The overall mortality observed was $18.8 \%$. In the late phases of the outbreak, there was a significant increase in disease severity without a statistically significant increase in mortality. Predictors of mortality on univariate analysis were age, diabetes mellitus, acute on chronic kidney disease, presence of symptoms on admission, NLR, CRP, LDH, computed tomography (CT) chest grades 3 and 4, and need for respiratory support; however, only age and the renal syndrome of acute on chronic kidney disease retained significance on multivariate analysis ( $p 0.003$ and $p 0.019$, respectively).

Conclusion Among patients on haemodialysis, higher mortality was observed in patients who were older, and among those with acute on chronic kidney disease. In the late phase of the outbreak, there was a statistically significant increase in disease severity without a corresponding increase in mortality.
\end{abstract}

Tanuj Moses Lamech

tanujlamech@gmail.com

1 Institute of Nephrology, Madras Medical College, Chennai,

India 


\section{Changing dynamics of Covid-19 in patients requiring haemodialysis: A single centre experience}

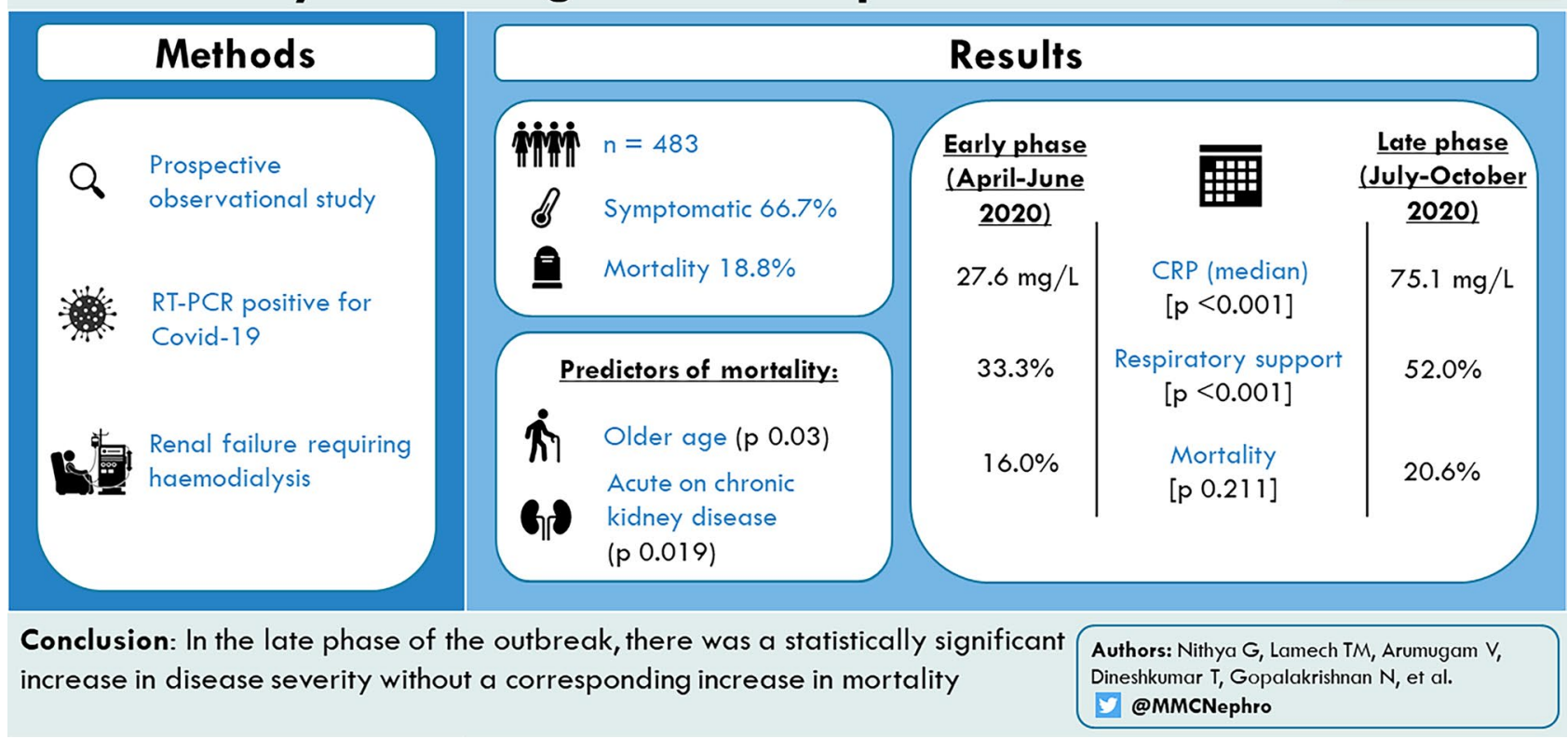

Keywords COVID-19 $\cdot$ Haemodialysis $\cdot$ Mortality $\cdot$ Remdesivir

\section{Introduction}

Corona Virus Disease 2019 (COVID-19) caused by the Severe Acute Respiratory Syndrome Coronavirus 2 (SARSCoV-2) was first identified in the Wuhan province of China, in December 2019 [1]. In the year that has since elapsed, the novel coronavirus has spread globally, and continues to place enormous strains on healthcare systems around the world. Patients with end-stage renal disease (ESRD) are at particularly high risk of both contracting the disease, and developing severe complications of the infection [2, 3]. In this observational study, we report our experience in managing patients with renal failure and COVID-19, along with the changes in clinical severity and patient outcomes that were observed over the course of the outbreak.

\section{Materials and methods}

This was a single centre prospective, observational study conducted at the Madras Medical College in Chennai, India, from April to October, 2020. The study was approved by the Institutional Ethics Committee. All patients with renal failure requiring haemodialysis (whatever the renal status before the outbreak) with COVID-19, as documented by a positive nasopharyngeal swab for SARS-CoV-2 by reversetranscriptase polymerase chain reaction (RT-PCR), were included in this study.

\section{Management protocols}

As per institutional policy, all patients with renal failure and COVID-19 were admitted irrespective of symptomatology. At admission, disease severity was categorised according to WHO classification as mild, moderate or severe based on room air oxygen saturations $\left(\mathrm{SpO}_{2}\right)$ of $>94 \%$, $90-94 \%,<90 \%$, respectively [4]. Initial evaluation included a complete haemogram with neutrophil-lymphocyte ratio (NLR), a basic metabolic profile (including serum glucose, creatinine, sodium and potassium), and serum markers of inflammation [including C-reactive protein (CRP) and lactate dehydrogenase (LDH)]. All patients who were assessed to be stable enough to be shifted for chest imaging, underwent computed tomography (CT) of the chest, which was reported as either being suggestive or not suggestive of COVID-19, based on the presence of subpleural groundglass opacities and consolidations. Among the films that 
were suggestive of COVID-19, the severity was graded based on the percentage of lung involvement (with Grades $1,2,3$ and 4 corresponding to $<25 \%, 26-50 \%, 51-75 \%$ and $>75 \%$ lung involvement, respectively).

Varying degrees of respiratory support were provided, based on clinical requirement, ranging from supplemental oxygen via simple face masks or non-rebreather masks, to more advanced respiratory support in the form of high flow nasal oxygen, continuous positive airway pressure, and occasionally tracheal intubation with mechanical ventilation. Patients requiring respiratory support were also given intravenous dexamethasone $8 \mathrm{mg}, \mathrm{q} 24 \mathrm{~h}$, for 10 days or until discharge, whichever was earlier, along with subcutaneous enoxaparin, $40 \mathrm{mg}$, q24h, for the duration of their hospitalisation. Enoxaparin was skipped on dialysis days, and patients were dialysed with standard anticoagulation (unfractionated heparin, $2500 \mathrm{U}$ bolus initially, followed by $750 \mathrm{U} / \mathrm{h})$.

Beginning in July 2020, patients with Grade 3 or 4 CT chest, and patients who were clinically deemed to be severely ill, were offered remdesivir as part of an expanded access programme, after informed consent. Safety data for remdesivir in the ESRD population was unavailable at the time, and therefore the institutional protocol was to administer a dose of $2.5 \mathrm{mg} / \mathrm{kg}$ of oedema-free body weight (dry weight) up to a maximum dose of $100 \mathrm{mg}, 4 \mathrm{~h}$ before each haemodialysis session, for a maximum of 6 doses.

Similarly, from August 2020, patients with Grade 3 or 4 CT chest, and patients who were clinically deemed to be severely ill, were also offered convalescent plasma therapy (200 ml of plasma infusions once or twice) based on availability. Convalescent plasma was collected from individuals with microbiologically proven COVID 19 (by RT-PCR for SARS-CoV-2) who had clinical recovery with detectable SARS-CoV-2 IgG antibodies in the serum at the time of donation. Plasma donation was done at least 28 days after the initial date of swab positivity.

All patients were assessed daily by a nephrologist, and haemodialysis schedules were as per clinical or biochemical indications and clinically determined volume status. Patients who required dialysis initiation were dialysed through temporary catheters placed into either the femoral or internal jugular veins. Patients were given intermittent haemodialysis at a separate dialysis unit, with single-use lines and dialysers. Continuous renal replacement therapy was not performed. All staff were provided with personal protective equipment, and were assigned in weekly rotations to exclusively attend to the COVID-19 dialysis unit.

Nasopharyngeal swabs for RT-PCR were repeated every $72 \mathrm{~h}$, until they turned negative. Discharge criteria included a single negative nasopharyngeal swab, clinical recovery, and the absence of respiratory support for at least $48 \mathrm{~h}$. Clinical outcomes were recorded, along with the physician-ascertained cause of death among deceased patients.

\section{Statistical methods}

Statistical analyses were performed using IBM ${ }^{\circledR}$ SPSS $^{\circledR}$ Statistics version 23. Categorical variables were expressed as number and proportion, normally distributed numerical variables were expressed as mean with standard deviation, and non-normally distributed variables were expressed as median with interquartile range. Appropriate tests of statistical significance were used, including the Chi-Square test for categorical data, the independent $t$-test for normally distributed data, and the Mann-Whitney $U$ test for non-normally distributed data. Predictors of mortality were analysed using the univariate and multivariate logistic regressions. A two-sided $p$ value $<0.05$ was considered to be statistically significant.

\section{Results}

\section{Baseline characteristics}

A total of 483 patients were included in this study, of whom $416(86.1 \%)$ had end-stage renal disease and were on maintenance haemodialysis. The mean age was $52( \pm 13.07)$ years, and $69.3 \%$ of them were male. The dialysis access was an AV fistula in 314 patients (65\%), a temporary HD catheter in 151 patients $(31.3 \%)$, a tunnelled catheter in 16 patients (3.3\%) and an $\mathrm{AV}$ graft in 2 patients $(0.4 \%)$. Among patients with CKD 5D who had a temporary HD catheter, the median dialysis vintage was 1 month (IQR 0.5-12). Chronic hepatitis B infection was present in 12 patients $(2.6 \%)$, chronic hepatitis $\mathrm{C}$ infection in 17 patients (3.5\%), HIV infection in 3 patients $(0.6 \%)$, and combined hepatitis $\mathrm{B}$ and $\mathrm{C}$ co-infection in 3 patients $(0.6 \%)$. The symptoms at presentation included fever in 217 patients (44.9\%), cough in 158 patients (32.7\%), and dyspnoea in 219 patients (45.3\%). Other baseline characteristics are described in Table 1.

\section{Comparisons between symptomatic and asymptomatic patients}

The mean age of patients who presented with symptoms was significantly higher than patients who were asymptomatic at presentation $(p=0.004)$. The percentage of patients with comorbidities was not statistically different between the two groups. Patients who were symptomatic at presentation were found to have significantly higher levels of NLR, CRP and LDH than those who were asymptomatic $(p<0.001)$, required more respiratory support $(p<0.001)$, and had higher degrees of lung involvement based on CT 
Table 1 Baseline characteristics and comparison between symptomatic and asymptomatic patients at presentation

\begin{tabular}{|c|c|c|c|c|}
\hline & Total cohort $(n=483)$ & $\begin{array}{l}\text { Symptomatic }(n=322) \\
(66.7 \%)\end{array}$ & $\begin{array}{l}\text { Asymptomatic } \\
(n=161)(33.4 \%)\end{array}$ & $p$ value \\
\hline Age (years; mean [SD]) & $52.16(13.07)$ & $53.01(12.77)$ & $50.47(13.53)$ & 0.004 \\
\hline Male/Female ratio & 2.26 & 2.35 & 2.1 & 0.577 \\
\hline \multicolumn{5}{|l|}{ Renal syndrome } \\
\hline CKD 5D $(n[\%])$ & $416(86.1)$ & $264(82)$ & $152(94.4)$ & \multirow[t]{4}{*}{0.001} \\
\hline CKD 5ND $(n[\%])$ & $15(3.1)$ & $12(3.7)$ & $3(1.9)$ & \\
\hline Acute on CKD $(n[\%])$ & $45(9.3)$ & $39(12.1)$ & $6(3.7)$ & \\
\hline $\mathrm{AKI}(n[\%])$ & $7(1.4)$ & $7(2.2)$ & 0 & \\
\hline \multicolumn{5}{|l|}{ Comorbidities } \\
\hline Diabetes mellitus ( $n[\%])$ & $194(40.6)$ & $132(41.4)$ & $62(39)$ & 0.617 \\
\hline Hypertension $(n[\%])$ & $384(80.3)$ & $262(82.1)$ & $122(76.7)$ & 0.162 \\
\hline Heart failure $(n[\%])$ & $81(16.9)$ & $51(16)$ & $30(18.9)$ & 0.429 \\
\hline Pulmonary pathologies $(n[\%])$ & $14(2.9)$ & $7(2.2)$ & $7(4.4)$ & 0.248 \\
\hline Dialysis vintage (months; median [IQR]) & $12(2-36)$ & $12(1-36)$ & $18(4-48)$ & 0.014 \\
\hline \multicolumn{5}{|l|}{ Laboratory parameters } \\
\hline NLR (median [IQR]) & $4.4(2.59-8.15)$ & $5.01(2.68-10.13)$ & $3.6(2.37-6.17)$ & $<0.001$ \\
\hline CRP (mg/L; median [IQR]) & $52(14.85-128.4)$ & $84(26-149.3)$ & $20.2(6.1-61.93)$ & $<0.001$ \\
\hline LDH (IU/L; median [IQR]) & $328.5(244-443)$ & $365(268-512)$ & $267(216-352)$ & $<0.001$ \\
\hline \multicolumn{5}{|l|}{ CT chest } \\
\hline Not suggestive $(n[\%])$ & $147(31.7)$ & $71(23.1)$ & $76(48.4)$ & \multirow[t]{5}{*}{$<0.001$} \\
\hline Grade $1(n[\%])$ & $131(28.2)$ & $72(23.5)$ & $59(37.6)$ & \\
\hline Grade $2(n[\%])$ & $89(19.2)$ & $75(24.4)$ & $14(8.9)$ & \\
\hline Grade $3(n[\%])$ & $63(13.6)$ & $58(18.9)$ & $5(3.2)$ & \\
\hline Grade $4(n[\%])$ & $34(7.3)$ & $31(10.1)$ & $3(1.9)$ & \\
\hline \multicolumn{5}{|l|}{ Clinical course } \\
\hline Respiratory support ( $n[\%])$ & $216(44.7)$ & $195(60.9)$ & $20(12.4)$ & $<0.001$ \\
\hline Duration of hospital stay (days; median [IQR]) & $8(5-11)$ & $8(6-11)$ & $6(5-10)$ & 0.002 \\
\hline Use of Remdesivir ( $n[\%])$ & $92(19)$ & $84(26.1)$ & $8(5)$ & $<0.001$ \\
\hline Use of convalescent plasma $(n[\%])$ & $31(6.4)$ & $29(9)$ & $2(1.2)$ & $\mathbf{0 . 0 0 1}$ \\
\hline Mortality $(n[\%])$ & $91(18.8)$ & $80(24.8)$ & $11(6.8)$ & $<0.001$ \\
\hline
\end{tabular}

NLR Neutrophil Lymphocyte Ratio, CRP C Reactive Protein, $L D H$ Lactate Dehydrogenase, CKD Chronic Kidney Disease, AKI Acute Kidney Injury

grade. Patients who were symptomatic at presentation were more likely to have received remdesivir $(p<0.001)$ and convalescent plasma $(p=0.001)$ and to have higher mortality $(p<0.001)$. The overall mortality in this study was $18.8 \%$.

\section{Comparisons between patients presenting in the first phase (April to June 2020) and second phase (July to October 2020) of the outbreak}

For the purposes of this analysis, the outbreak was divided into two phases-a first phase from April to June 2020, which included 187 patients, and a second phase from July to October 2020, which included 296 patients (Table 2). Patients in the second phase had a significantly higher mean age $(p=0.03)$, and were more likely to; have symptoms at presentation $(p<0.001)$, present with acute on chronic kidney disease $(p=0.007)$, have comorbid conditions such as diabetes mellitus $(p=0.014)$, heart failure $(p<0.001)$ or pre-existing lung disease $(p=0.002)$, and have a temporary haemodialysis catheter as their dialysis access $(p=0.05)$. Inflammatory markers including NLR, CRP and LDH were significantly higher in patients presenting in this second phase of the outbreak ( $p<0.001,<0.001,<0.001$, respectively), and patients were more likely to have higher grades on CT chest $(p<0.001)$ and to require respiratory support at admission $(p<0.001)$ (Fig. 1).

Despite these demonstrable increases in disease severity, there was no statistically significant difference in mortality between patients admitted in phase 1 and phase 2 of the outbreak ( $p=0.211)$. This was true even when mortality was stratified according to disease severity. 
Table 2 Comparisons between patients presenting in the first phase (April to June 2020) and second phase (July to October 2020) of the outbreak

\begin{tabular}{|c|c|c|c|}
\hline & April to June $(n=187)$ & July to October $(n=296)$ & $p$ value \\
\hline Age (years; mean [SD]) & $50.53(12.88)$ & $53.19(13.1)$ & 0.03 \\
\hline Male/Female ratio & 1.83 & 2.6 & 0.078 \\
\hline \multicolumn{4}{|l|}{ Renal syndrome } \\
\hline CKD 5D $(n[\%])$ & $173(92.5)$ & $243(82.1)$ & \multirow[t]{4}{*}{$\mathbf{0 . 0 0 7}$} \\
\hline CKD 5ND $(n[\%])$ & $5(2.7)$ & $10(3.4)$ & \\
\hline Acute on CKD $(n[\%])$ & $8(4.3)$ & $37(12.5)$ & \\
\hline $\mathrm{AKI}(n[\%])$ & $1(0.5)$ & $6(2)$ & \\
\hline \multicolumn{4}{|l|}{ Comorbidities } \\
\hline Diabetes mellitus ( $n[\%])$ & $63(33.7)$ & $131(45)$ & 0.014 \\
\hline Hypertension $(n[\%])$ & $144(77)$ & $240(82.5)$ & 0.142 \\
\hline Heart failure $(n[\%])$ & $46(24.6)$ & $35(12)$ & $<0.001$ \\
\hline Pulmonary pathologies $(n[\%])$ & $11(5.9)$ & $3(1)$ & 0.002 \\
\hline Dialysis vintage (months; median [IQR]) & $18(6-36)$ & $12(0.5-36)$ & 0.004 \\
\hline Symptoms $(n[\%])$ & $97(51.9)$ & $225(76)$ & $<0.001$ \\
\hline Fever $(n[\%])$ & $85(45.5)$ & $132(44.6)$ & 0.853 \\
\hline Cough $(n[\%])$ & $66(35.3)$ & $91(31.1)$ & 0.336 \\
\hline Dyspnoea $(n[\%])$ & $62(33.2)$ & $157(53)$ & $<0.001$ \\
\hline \multicolumn{4}{|l|}{ Laboratory parameters } \\
\hline NLR (median [IQR]) & $3.2(2.25-6.06)$ & $5.6(3-10.2)$ & $<0.001$ \\
\hline CRP (mg/L; median [IQR]) & $27.6(7.3-98.7)$ & $75.1(22.8-149.25)$ & $<0.001$ \\
\hline LDH (IU/L; median [IQR]) & 289 (209-389) & $354(264.75-514.5)$ & $<0.001$ \\
\hline \multicolumn{4}{|l|}{ CT chest } \\
\hline Not suggestive (n [\%]) & $82(44.3)$ & $65(23.3)$ & \multirow[t]{5}{*}{$<0.001$} \\
\hline Grade $1(n[\%])$ & $50(27)$ & $81(29)$ & \\
\hline Grade $2(n[\%])$ & $29(15.7)$ & $60(21.5)$ & \\
\hline Grade 3 ( $n[\%])$ & $16(8.6)$ & $47(16.8)$ & \\
\hline Grade $4(n[\%])$ & $8(4.3)$ & $26(9.3)$ & \\
\hline \multicolumn{4}{|l|}{ Clinical presentation } \\
\hline Mild (n [\%]) & $136(72.7)$ & $164(55.4)$ & \multirow[t]{3}{*}{$<0.001$} \\
\hline Moderate $(n[\%])$ & $31(11.2)$ & 65 (21.9) & \\
\hline Severe $(n[\%])$ & $20(10.7)$ & $67(22.6)$ & \\
\hline \multicolumn{4}{|l|}{ Clinical course } \\
\hline $\begin{array}{l}\text { Respiratory support } \\
(n[\%])\end{array}$ & $62(33.3)$ & $154(52)$ & $<0.001$ \\
\hline Duration of hospital stay (days; median [IQR]) & $8(6-11)$ & $7(5-11)$ & 0.543 \\
\hline Total mortality $(n[\%])$ & $30(16)$ & $61(20.6)$ & 0.211 \\
\hline \multicolumn{4}{|l|}{ Stratification of mortality according to severity } \\
\hline Mild disease $(n[\%])$ & $12(8.8)$ & $15(9.1)$ & 0.924 \\
\hline Moderate disease $(n[\%])$ & $8(25.8)$ & $18(27.7)$ & 0.846 \\
\hline Severe disease $(n[\%])$ & $10(50)$ & $28(41.8)$ & 0.516 \\
\hline
\end{tabular}

NLR Neutrophil Lymphocyte Ratio, CRP C Reactive Protein, $L D H$ Lactate Dehydrogenase, $C K D$ Chronic Kidney Disease, AKI Acute Kidney Injury

\section{Predictors of mortality}

On univariate analysis (Table 3), the statistically significant predictors of mortality were age (OR, $1.052[95 \% \mathrm{Cl}$, 1.031-1.073]; $p<0.001$ ), diabetes mellitus (OR, 2.003 [95\% Cl, 1.261-3.182]; $p=0.004)$, acute on chronic kidney disease (OR, 5.689 [95\% Cl, 3.002-10.779]; $p<0.001$ ), presence of symptoms at presentation (OR, 4.508 [95\% $\mathrm{Cl}, 2.324-8.742] ; p<0.001)$, moderate and severe disease [(OR, 1.834 [95\% Cl, 1.091-3.105]; $p=0.021)$ and (OR, 5.019 [95\% Cl, 3.005-8.383]; $p<0.001$ ), respectively], NLR (OR, 1.052 [95\% Cl, 1.026-1.080]; $p<0.001)$, CRP (OR, 


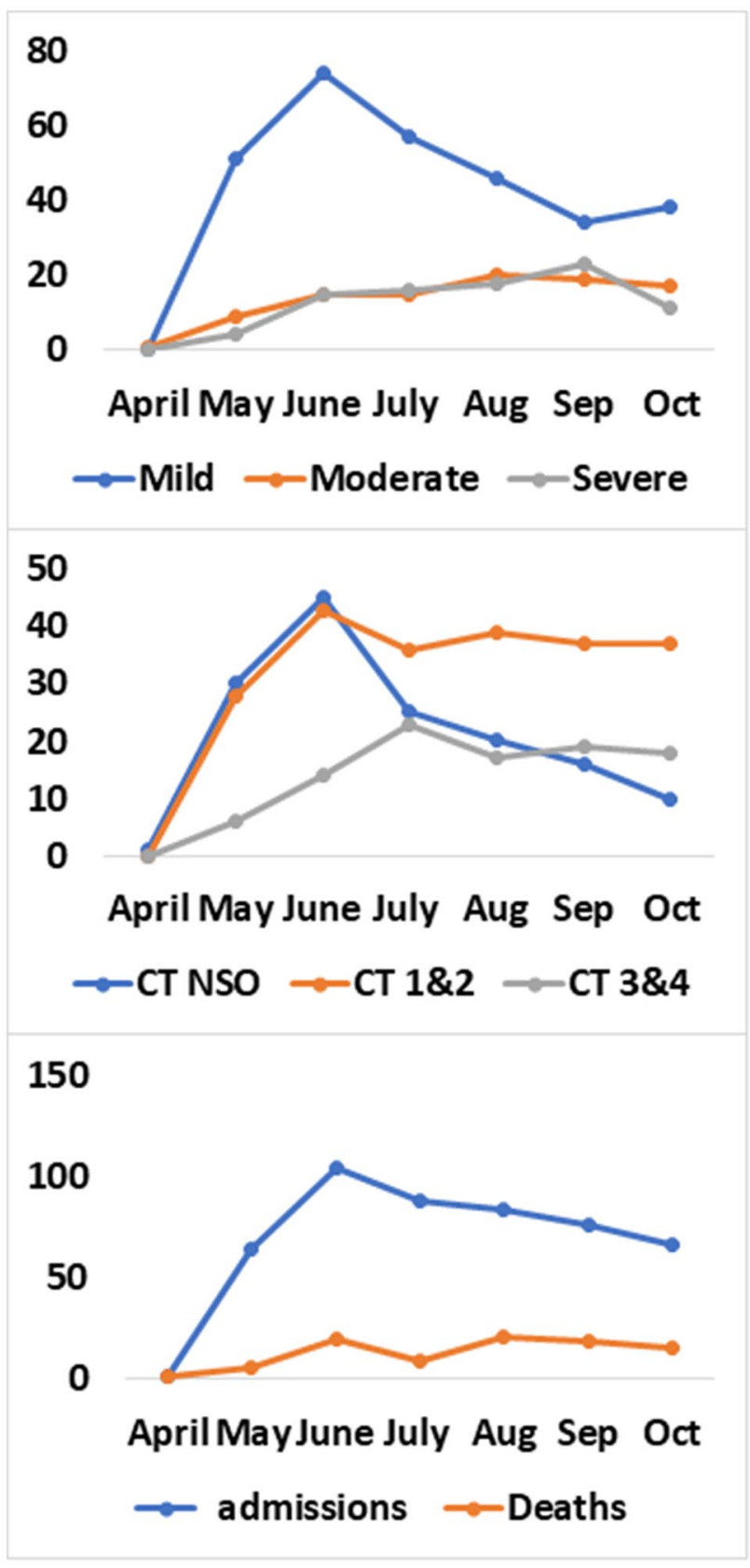

Fig. 1 Changing dynamics in the severity of presentation, CT chest, admissions and mortality. (CT NSO-CT not suggestive of COVID19; CT 1 and 2-CT chest grades 1 and 2; CT 3 and 4-CT chest grades 3 and 4 )

1.006 [95\% Cl, 1.004-1.008]; $p<0.001)$, LDH (OR, 1.003 [95\% Cl, 1.002-1.004]; $p<0.001$ ), CT grades 3 and 4 (OR, $5.922[95 \% \mathrm{Cl}, 3.474-10.095] ; p<0.001)$ and requirement for respiratory support (OR, 6.526 [95\% Cl, 3.782-11.262]; $p<0.001)$.

When a multivariate analysis was performed, by including in the model only those predictors that were statistically significant on univariate analysis, only age (OR, $1.044[95 \%$
$\mathrm{Cl}, 1.015-1.075] ; p=0.003)$ and presentation as acute on chronic kidney disease (OR, 2.709 [95\% Cl, 1.111-6.6.03]; $p=0.028)$ retained their statistical significance.

\section{Effect of remdesivir and convalescent plasma on mortality}

The use of remdesivir and convalescent plasma did not have any significant effect on mortality, irrespective of clinical severity (Table 4).

\section{Cause of death analysis}

A total of 91 patients died during hospitalisation. The most common cause of death was respiratory failure $(49.5 \%)$. Other causes include encephalopathy of unknown cause (9.9\%), septic shock (9.9\%), uraemic encephalopathy (3.3\%), pulmonary oedema (3.3\%), diabetic ketoacidosis $(3.3 \%)$, septic encephalopathy $(2.2 \%)$, sudden cardiac death $(2.2 \%)$, brainstem dysfunction due to massive cerebral infarct (2.2\%), AV Fistula rupture (1.1\%), suicide (1.1\%), and undetermined cause (12.1\%).

\section{Discussion}

While much has been written about COVID-19 over the last year, outcome data in patients with ESRD remain confined to small case series. This single-centre experience of handling patients on dialysis adds to the limited literature regarding the management of COVID-19 in patients with ESRD. Several changes occurred in both clinical severity and therapeutic intervention, as the outbreak waxed and eventually waned.

In the early phase of the outbreak, the FDA recommendation to avoid the use of remdesivir in patients with an eGFR $<30 \mathrm{~mL} / \mathrm{min} / 1.73 \mathrm{~m}^{2}$ resulted in this potentially beneficial agent being denied to individuals who might have most benefited from it [5]. The safety of remdesivir in the dialysis population has since been demonstrated putting an end to the bleak 'renalism' that accompanied its original restricted use [6]. Nevertheless, our analysis failed to identify any mortality benefit of the drug.

As the outbreak progressed, the patients admitted to our institution were demonstrably more severely ill, requiring greater degrees of respiratory support, with a greater proportion receiving remdesivir therapy and convalescent plasma. Interestingly, the mortality rates did not show a proportional increase, despite the increase in disease severity. This may perhaps be explained by improved quality of care, resulting both from logistic reasons such as streamlining of emergency room care and better bed management, and from medical reasons such as standardisation of therapy 
Table 3 Predictors of mortality by univariate analysis

\begin{tabular}{lcclr}
\hline & Dead $(n=91)$ & Survived $(n=392)$ & Odds ratio & $p$ value \\
& & & OR $(95 \%$ CI $)$ & \\
\hline Age (years; mean [SD]) & $58.4(11.68)$ & $50.7(12.95)$ & $1.052(1.031-1.073)$ & $<\mathbf{0 . 0 0 1}$ \\
Diabetes mellitus $(n[\%])$ & $49(54.4)$ & $145(37.4)$ & $2.003(1.261-3.182)$ & $\mathbf{0 . 0 0 4}$ \\
Heart failure $(n[\%])$ & $15(16.7)$ & $66(17)$ & $0.976(0.528-1.804)$ & 0.938 \\
Acute on CKD $(n[\%])$ & $23(25.3)$ & $22(5.6)$ & $5.689(3.002-10.779)$ & $<\mathbf{0 . 0 0 1}$ \\
Symptoms $(n[\%])$ & $80(87.9)$ & $242(61.7)$ & $4.508(2.324-8.742$ & $<\mathbf{0 . 0 0 1}$ \\
Mild disease $(n[\%])$ & $27(29.7)$ & $273(69.6)$ & $0.184(0.112-0.303)$ & $<\mathbf{0 . 0 0 1}$ \\
Moderate disease $(n[\%])$ & $26(28.6)$ & $70(17.9)$ & $1.834(1.091-3.105)$ & $\mathbf{0 . 0 2 1}$ \\
Severe disease $(n[\%])$ & $38(41.8)$ & $49(12.5)$ & $5.019(3.005-8.383)$ & $<\mathbf{0 . 0 0 1}$ \\
NLR (median [IQR]) & $7.75(3.8-17.17)$ & $3.95(2.35-7.23)$ & $1.052(1.026-1.080)$ & $<\mathbf{0 . 0 0 1}$ \\
CRP (mg/L; median $[\mathrm{IQR}])$ & $128.8(72.2-182.1)$ & $36.15(12-105.52)$ & $1.006(1.004-1.008)$ & $<\mathbf{0 . 0 0 1}$ \\
LDH (IU/L; median [IQR]) & $431(319-643)$ & $307(235-409)$ & $1.003(1.002-1.004)$ & $<\mathbf{0 . 0 0 1}$ \\
CT Not suggestive $(n[\%])$ & $11(14.9)$ & $136(34.9)$ & $0.326(0.166-0.639)$ & $\mathbf{0 . 0 0 1}$ \\
CT Grade 3 and 4 $(n[\%])$ & $25(33.8)$ & $59(15.1)$ & $5.922(3.474-10.095)$ & $<\mathbf{0 . 0 0 1}$ \\
Respiratory support $(n[\%])$ & $72(79.1)$ & $144(36.7)$ & $6.526(3.782-11.262)$ & $<\mathbf{0 . 0 0 1}$ \\
\hline
\end{tabular}

NLR Neutrophil Lymphocyte Ratio, $C R P$ C Reactive Protein, $L D H$ Lactate Dehydrogenase, $C K D$ Chronic Kidney Disease

\begin{tabular}{lcclc}
\hline & Dead $n(\%)$ & Survived $n(\%)$ & Odds ratio OR (95\% CI) & $p$ value \\
\hline Severe presentation & & & & \\
$\quad$ Remdesivir & $14(37.8)$ & $23(62.2)$ & $0.659(0.277-1.567)$ & 0.345 \\
$\quad$ Convalescent plasma & $6(15.8)$ & $6(12.2)$ & $1.344(0.396-9.554)$ & 0.635 \\
Moderate presentation & & & & \\
Remdesivir & $8(26.7)$ & $22(73.3)$ & $0.970(0.366-2.568)$ & 0.951 \\
$\quad$ Convalescent plasma & $6(23.1)$ & $7(10)$ & $2.700(0.813-8.972)$ & 0.105 \\
Mild presentation & & & & \\
$\quad$ Remdesivir & $3(12)$ & $22(88)$ & $1.426(0.398-5.113)$ & 0.481 \\
Convalescent plasma & $2(7.4)$ & $4(1.5)$ & $5.380(0.939-30.841)$ & 0.059 \\
\hline
\end{tabular}

Table 4 Effect of the use of remdesivir and convalescent plasma on mortality encephalopathy with no identifiable cause, potentially implicating COVID-19 encephalitis. In keeping with existing literature indicating that COVID-19 predisposes to complications such as hyperglycaemic crises, hypercoagulability, and psychiatric disturbances, $3.3 \%, 2.2 \%$ and $1.1 \%$ of deaths were attributable to diabetic ketoacidosis, ischaemic stroke, and even suicide, respectively.

\section{Strengths of the study}

Being a tertiary care centre, patients admitted to our institution were referred from across a wide geographical area for COVID care services. Thus, these findings may be extrapolatable to a population level, at least for South India.

\section{Limitations of the study}

Because of the absence of an electronic health record and the circumstances of the pandemic, data collection was of death. However, $9.9 \%$ of deaths were preceded by 
performed according to a prespecified protocol, resulting in the loss of some granularity. Patients who succumbed before they could be dialysed were not included in our analysis, potentially resulting in some selection bias.

\section{Conclusions}

Among patients on haemodialysis, higher mortality was observed in those who were older and in individuals who presented with acute on chronic kidney disease requiring dialysis. In the late phase of the outbreak, there was a statistically significant increase in disease severity, with no parallel increase in mortality.

Supplementary Information The online version contains supplementary material available at https://doi.org/10.1007/s40620-021-01072-4.

Acknowledgements We gratefully acknowledge the contributions of all of the staff of the Institute of Nephrology, and especially our dialysis technicians, without whom this endeavour would never have come to fruition.

Funding The authors did not receive support from any organization for the submitted work.

Data availability The datasets generated and/or analysed during the current study are available from the corresponding author on reasonable request.

\section{Declarations}

Conflict of interest The authors have no relevant financial or non-financial interests to disclose.

Ethical approval Obtained from the Institutional Ethics Committee, Madras Medical College.

Consent to participate Verbal informed consent was obtained prior to the interview.

\section{References}

1. Huang C, Wang Y, Li X et al (2020) Clinical features of patients infected with 2019 novel coronavirus in Wuhan, China. Lancet 395(10223):497-506
2. D'Marco L, Puchades MJ, Romero-Parra M et al (2020) Coronavirus disease 2019 in chronic kidney disease. Clin Kidney J 13(3):297-306

3. Couchoud C, Bayer F, Ayav C et al (2020) Low incidence of SARS-CoV-2, risk factors of mortality and the course of illness in the French national cohort of dialysis patients. Kidney Int 98(6): 1519-1529

4. World Health Organization (2020) Clinical management of COVID-19: interim guidance, 27 May 2020. [Internet]. https:// apps.who.int/iris/handle/10665/332196. Accessed 12 Feb 2021

5. Fact sheet for health care providers Emergency Use Authorization (EUA) of Veklury (remdesivir). [Internet]. https://www.fda.gov/ media/143189/download. Accessed 12 Feb 2021

6. Aiswarya D, Arumugam V, Dineshkumar T et al (2020) Use of remdesivir in patients with COVID-19 on hemodialysis: a study of safety and tolerance. Kidney Int Rep. https://doi.org/10.1016/j. ekir.2020.12.003

7. WHO Coronavirus Disease (COVID-19) Dashboard. [Internet]. https://covid19.who.int. Accessed 16 Feb 2021

8. Milia VL, Bacchini G, Bigi MC et al (2020) COVID-19 outbreak in a large hemodialysis center in Lombardy, Italy. Kidney Int Rep 5(7):1095-1099

9. Wang $\mathrm{R}, \mathrm{He} \mathrm{H}$, Liao $\mathrm{C}$ et al (2020) Clinical outcomes of hemodialysis patients infected with severe acute respiratory syndrome coronavirus 2 and impact of proactive chest computed tomography scans. Clin Kidney J 13(3):328-333

10. Ng JH, Hirsch JS, Wanchoo R et al (2020) Outcomes of patients with end-stage kidney disease hospitalized with COVID-19. Kidney Int 98(6):1530-1539

11. Lano G, Braconnier A, Bataille S et al (2020) Risk factors for severity of COVID-19 in chronic dialysis patients from a multicentre French cohort. Clin Kidney J 13(5):878-888

12. Hilbrands LB, Duivenvoorden R, Vart P et al (2020) COVID19-related mortality in kidney transplant and dialysis patients: results of the ERACODA collaboration. Nephrol Dial Transplant 35(11):1973-1983

13. Ozturk S, Turgutalp K, Arici M et al (2020) Mortality analysis of COVID-19 infection in chronic kidney disease, haemodialysis and renal transplant patients compared with patients without kidney disease: a nationwide analysis from Turkey. Nephrol Dial Transplant 35(12):2083-2095

Publisher's Note Springer Nature remains neutral with regard to jurisdictional claims in published maps and institutional affiliations. 